\title{
RECURSOS TECNOLÓGICOS PARA EL APRENDIZAJE MÓVIL (MLEARNING) Y SU RELACIÓN CON LOS AMBIENTES DE EDUCACIÓN A DISTANCIA: IMPLEMENTACIONES E INVESTIGACIONES
}

\author{
(MOBILE LEARNING -MLEARNING- TECHNOLOGY RESOURCES AND THEIR RELATIONSHIP \\ WITH DISTANCE LEARNING ENVIRONMENTS: APPLICATIONS AND RESEARCH STUDIES)
}

\author{
María Soledad Ramírez Montoya \\ Tecnológico de Monterrey, ITESM (México)
}

\section{RESUMEN}

El artículo tiene por objetivo describir la experiencia de la implementación de recursos tecnológicos para el aprendizaje móvil (mlearning) en ambientes educativos, colocando especial énfasis en los ambientes a distancia, con el fin de analizar sus implicaciones operativas y los retos que expone este tipo de innovaciones educativas. El tema presenta conceptualizaciones teóricas, implementaciones prácticas de aprendizaje móvil en ambientes a distancia y en ambientes multimodales, así como estudios que se han desarrollado en estas implementaciones. Las implicaciones operativas detectadas son: (1) de orden tecnológico, (2) del diseño de la configuración tecnológica, y (3) de integración en ambientes de aprendizaje. Los retos que se vislumbran son los relacionados con: (1) el soporte tecnológico de las redes inalámbricas, (2) la capacidad de almacenamiento en dispositivos móviles, y (3) la configuración de contenido de los recursos.

Palabras clave: aprendizaje móvil, recursos tecnológicos, dispositivos móviles, ambientes a distancia, investigación de tecnologías educativas.

\begin{abstract}
This article describes the implementation of technology resources in mobile learning (mlearning) environments, with particular emphasis on distance education environments. The purpose is to analyze implications and challenges related to the implementation of these educative innovations. This paper
\end{abstract}


presents the theoretical conceptualizations and discusses practical implementation of mlearning in multimodal distance learning settings. Operative implications discussed are: (1) the technology sequence to follow, (2) design of the technology set up; and (3) integration of diverse learning environments. Challenges that must be addressed include: (1) support forwireless networks, (2) storage capacity for mobile devices, and (3) the set up of the content of the resources.

Keywords: mobile learning, technology resources, mobile devices, distance learning environments, research on educational technologies.

\section{DE INTERNET A LOS RECURSOS MEDIADOS POR TECNOLOGÍA A TRAVÉS DE LOS DISPOSITIVOS MÓVILES}

Los avances en ciencia y tecnología han traído consigo un cambio sustancial en las prácticas de todas las esferas de la sociedad. Desde aquel 30 de abril de $1993 \mathrm{el}$ Laboratorio Europeo de Física de Partículas (CERN), con sede en Ginebra, anunció la disponibilidad pública de un programa informático llamando World Wide Web (WWW). Es decir, el inicio del Internet como una herramienta pública, que en poco más de una década ha transformado de forma radical muchas de las actividades de las personas a lo largo del mundo. La idea inicial era facilitar la comunicación entre los científicos del CERN y sus colegas de otros países basándose en el uso de los equipos de cómputo.

Para llegar a lo que hoy es el Internet hicieron falta muchos desarrollos tecnológicos, entre ellos, el programa informático que permitiera almacenar información partiendo del concepto de hipertexto. Su creador fue el británico Tim Bernrs-Lee nacido en 1955, investigador del CERN y el Enquire, como se le llamó, que es en realidad un rudimentario navegador. Posteriormente se llevó a cabo el proyecto Hyper-Text basado en el lenguaje HTML (Hiper-Text Mark -up Language) que es la lengua de la Web y que propició que su uso se extendiese (Beners- Lee, 1999).

Otros desarrollos tecnológicos tales como el diseño y establecimiento de protocolos, la tecnología de interconexión y los servicios de la accesibilidad, han hecho posible el universo virtual de comunicación: la Red de redes. La conmutación de datos por paquetes, que dio pie a la Arponet (por sus siglas en inglés Advanced Research Project Agency Network) un proyecto del Departamento de defensa Estadounidense, que permitía el intercambio de información a través de la red de computadoras. Se puede decir que es la primera gran red mundial de intercambio de información por paquetes y que después llegaría a ser el Internet (De Pablos, 
1998). La Red ha tenido impacto en muchas de las actividades de información y de comunicación entre las personas. El ámbito educativo no ha quedado al margen de esta influencia.

En la educación hemos sido testigos de que los recursos didácticos mediados por tecnologías son una alternativa para desarrollar procesos de aprendizaje. La implementación de estos medios ha generado el diseño de diversos ambientes, más allá de los presenciales y es así como es común ahora encontrar que, con el apoyo de estos recursos tecnológicos (con mayor o menor uso), se habla de ambientes multimodales, ambientes combinados o mixtos (blended learning, b-Learning, Young, 2002), ambientes digitales en línea por Internet (electronic learning, eLearning, Pastore, 2002) y, recientemente, ambientes de aprendizaje móvil (mobile learning, m-learning, Laouris, 2005).

En esta línea, el objetivo de este artículo es describir la experiencia de la implementación de recursos tecnológicos para el aprendizaje móvil (mlearning) en ambientes educativos, colocando especial énfasis en los ambientes a distancia, con el fin de analizar sus implicaciones operativas y los retos que expone este tipo de innovaciones educativas. El tema es presentado primero desde una conceptualización teórica, mencionando las características de los dispositivos móviles y las redes que posibilitan su uso, en un segundo momento se aborda la experiencia práctica de una institución de educación superior, describiendo sus implementaciones en ambientes multimodales y educación a distancia, posteriormente se describen sus implementaciones en la puesta en práctica, los desarrollos tecnológicos que se han desarrollado, las investigaciones que indagan sus procesos y el artículo cierra con unas reflexiones que consideran las ventajas de su uso y los retos que se vislumbran en la integración de estas innovaciones.

\section{EL APRENDIZAJE MÓVIL DESDE LAS CONCEPTUALIZACIONES TEÓRICAS}

\section{Definiciones conceptuales de "aprendizaje móvil"}

La incursión de los recursos tecnológicos que apoyan los procesos de aprendizaje móvil han tenido influencias paralelas desde diversas vertientes, por un lado se encuentra el desarrollo de los servicios de comunicación, la facilidad de acceso a la información por las redes inalámbricas, la cantidad de dispositivos móviles que aparecen en el mercado y hasta las prácticas de la sociedad actual donde la 
movilidad, los tiempos de traslados y "esperas" han provocado la opción de "recursos para la productividad". Estos hechos han dado pie para que se hagan estudios sobre movilidad y prácticas, uno de estos fue el realizado por Schrank y Lomax (2002) desde el Instituto de Transporte de Texas de la Universidad de Texas A\&M donde se vio reflejada la cantidad de tiempos invertidos de los sujetos, en los traslados para el trabajo.

La capitalización de los tiempos y la movilidad se ponen en perspectiva, no sólo en lo que implica para una persona, sino también para una organización o para cualquier país que quiera aprovechar esta coyuntura. Estas temáticas han sido unidas a conceptos del aprendizaje a lo largo de la vida, desempeño organizacional, recursos de aprendizaje, lo que han llevado a pensar en el aprendizaje móvil (abreviado como m-learning, en el habla anglosajona) como una oportunidad más para seguir aprendiendo. El m-learning tiene fuerza principalmente en Europa (con desarrolladores líderes en recursos Ericsson y Nokia) y en Estados Unidos (con aplicaciones para asistentes personales digitales -PDA-) (Clyde, 2004).

El aprendizaje móvil tiene varias definiciones, dependiendo del enfoque donde se ubica dentro de los ambientes de aprendizaje:

- Elm-learning es el descendiente directo del $e$-learning para varios investigadores (Pinkwart, Hoppe, Milrad y Pérez, 2003; Quinn, 2000), dado que el $e$-learning es el aprendizaje apoyado por recursos y herramientas electrónicas digitales y $m$ learning es el e-learning que se apoya de dispositivos móviles y transmisión de wireless; o simplemente, es cuando el aprendizaje toma lugar con dispositivos móviles.

- En contraparte, Sharples (2005) describe el aprendizaje como un proceso de acercamiento al conocimiento, donde los participantes en cooperación con sus compañeros y profesores, construyen en forma conjunta la interpretación de su mundo. Esta definición da a las tecnologías móviles un rol especial porque incrementa sus posibilidades de comunicación y conversación.

- Salz (2005) menciona que es el que se da a través de enseñanzas que no están limitadas por el ambiente de aprendizaje, sino que lo complementa, enriquece y estimula para provocar un aprendizaje flexible y móvil, que le ayuda al estudiante a aprender desde diferentes escenarios y contextos.

- mlearning es una manera de apoyar al aprendizaje en un medio ambiente donde diversos elementos como la espontaneidad, la personalización, la informalidad, 
la contextualización, la portabilidad, la conveniencia, la adaptabilidad, la integración y la disponibilidad, juegan un papel relevante (Laouris \& Eteokleous, 2005).

- Otros investigadores (Grupo de e-learning 360, citado por Quinn, 2007) lo definen más a partir del proceso y mencionan que el m-learning es cualquier actividad que permite a los individuos ser más productivos cuando consumen, interactúan con o crean información, mediada a través de un dispositivo digital compacto, que el individuo lleva consigo de manera constante, que tiene una conectividad confiable y que le cabe en el bolsillo.

Estas cinco delimitaciones dan luz sobre las diferentes formas en que puede ser definido el término de aprendizaje móvil, pero en ellas podemos vislumbrar concordancia en que intervienen dos elementos: movimiento y aprendizaje. Estos dos elementos son transmitidos a través de ciertos recursos: los dispositivos móviles.

\section{Dispositivos móviles}

Un dispositivo móvil es un procesador con memoria que tiene muchas formas de entrada (teclado, pantalla, botones, etc.), también formas de salida (texto, gráficas, pantalla, vibración, audio, cable). Algunos dispositivos móviles ligados al aprendizaje son las laptops, teléfonos celulares, teléfonos inteligentes, asistentes personales digitales (Personal Digital Assistant, PDA, por sus siglas en inglés), reproductores de audio portátil, iPods, relojes con conexión, plataforma de juegos, etc.; conectados a Internet, o no necesariamente conectados (cuando ya se han "archivado" los materiales).

Estos dispositivos funcionan a través de conexiones inalámbricas, algunas de ellas son presentadas por Metcalf (2006):

- Wide Area Network (WAN, por sus siglas en inglés) que puede darle una cobertura nacional e internacional a los celulares; los dispositivos en estas categorías son los smart phones, dispositivos de celulares, módems de celulares y sistemas satelitales.

- Local Area Network (LAN por sus siglas en inglés) es un esquema de conexión de red, tiene la capacidad de conectarse al internet sin cables unidos a una pared a través de un servidor de internet (por ejemplo un proxy server). Por medio de $W i F i$ las computadoras pueden enviar y recibir comunicación en cualquier 
parte, como una estación local y operar con la misma rapidez que las conexiones de modem.

- Metropolitan Area Networks (MANs, por sus siglas en inglés) pueden ser más difíciles de categorizar que las WANs o LANs, aquí intervienen muchas tecnologías que pueden cubrir una región más pequeña que un país o una geografía, pero puede ser más amplia que un edificio o un campus, el área de cobertura puede sobrepasar un edificio, pero no envolver una región más grande que una ciudad y para ello se apoyan en algunos "candados" como tecnologías combinadas (televisión por cable o recepción de wireless, por ejemplo), láser y sistemas microcelulares.

- Personal Area Network (PAN, por sus siglas en inglés) es la capacidad que se puede tener para conectar un dispositivo con otro por medio de una red personal, por ejemplo, un teléfono celular puede conectarse por la tecnología de Bluetooth (comunicación infrarroja) para enviar datos a otro celular o a una laptop, otro ejemplo puede darse en la interconexión de un teléfono celular usado como un modem vía Bluetooth para proporcionar conexión de internet.

Con esta visión podemos ver cómo las comunicaciones de redes inalámbricas, sus tendencias y productos están revolucionando (e incrementando en el día a día) el cómo nos comunicamos y las posibilidades de acceso que tenemos. Aún así, es conveniente también señalar que las redes móviles, al ser nodos móviles, pueden tener variaciones y los enlaces entre nodos (ya sean unidireccionales o bidireccionales) se pueden interrumpir, aunado a esto tenemos que tener presente que el ancho de banda disponible en una interface inalámbrica es inferior al que utiliza cables, y además pueden darse interferencias en las señales electromagnéticas.

En este abanico de posibilidades (y de retos, también), Kukulska-Hulme y Traxler (2005) mencionan que los recursos a través de dispositivos móviles pueden aportar al ámbito de los procesos de enseñanza y aprendizaje muchas potencialidades y que se requieren concebir nuevos métodos, prácticas y diseños que contemplen las características tecnológicas particulares que tienen los dispositivos. Estas características se dan por la esencia de la "portabilidad" del dispositivo, pero esto también puede llegar a ser una potencialidad por: la posibilidad de conexión para comunicaciones espontáneas y colaborativas, capacidad de proveer información de dispositivo a dispositivo, localización de información inmediata, capacidad de recursos con sonido, grabación, cámaras, videoclips. 


\section{Recursos mediados por tecnología}

Algunos recursos y aplicaciones que se encuentran disponibles y susceptibles de ser integrados en los ambientes de aprendizaje son los siguientes: Blogs, sistemas de administración de cursos, mensajes instantáneos, Wikis, Podcast, RSS (Really Simple Syndication, RSS por sus siglas en inglés), espacios sociales y otras herramientas de Web (Richardson, 2006; Green, Brown y Robinson, 2008). Es importante recordar la rapidez que se tiene para los cambios tecnológicos y que, en los siguientes años, este listado podría llegar a ser muy diferente.

Estos recursos tecnológicos están siendo integrados en los ambientes de aprendizaje a distancia, multimodales, combinados o de mlearning. El uso y las posibilidades que pueda hacerse de ellos están en relación directa con los aprendizajes que se quieran promover y para ello la creatividad en el diseño juega un papel importante, así como las condiciones de implementación que se realicen para que sea integrado en estos ambientes. Kenning (2007) pone en relieve que las innovaciones tecnológicas requieren ambientes receptivos. La percepción de los atributos de las innovaciones puede brindar beneficios que sean visto y alineados con otras tendencias sociales y expectativas. De la misma manera, Warschauer (2003) menciona que si estas innovaciones no son acompañadas de programas educativos bien fundamentados y tratados, no va a ir más allá de brindarle a la gente "nuevos juguetes" a través de tecnologías de alta definición. De ahí la importancia de considerar que las incorporaciones de innovaciones tecnológicas en los ambientes de aprendizaje deben ir acompañadas de planes estratégicos, para que los usuarios se "apropien" de estas nuevas tecnologías.

La apropiación tecnológica es un término que ha empezado a difundirse en publicaciones académicas a partir del año 2000, principalmente en países como Australia y Estados Unidos. El término surge de las áreas de ciencias computacionales y sistemas de información y se está aplicando recientemente a contextos educativos. Apunta a que los usuarios de tecnología "se adueñan", "hacen propia" la tecnología en la medida que la adoptan y adaptan a sus capacidades. Si bien el término ha sido desarrollado por autores que han trabajado el fenómeno en aplicaciones con computadoras (Urrea, 2006; Montes y Ochoa, 2006 y Morales, Monje y Loyola, 2006), se puede considerar que lo que visualizan puede ser fácilmente aplicable cuando se usan recursos tecnológicos para el aprendizaje a través de dispositivos móviles. Estos autores hablan de tres grandes etapas por las que el sujeto incursiona: (1) apropiación del objeto, la tecnología es considerada en forma muy superficial, sin realmente pensar acerca de sus beneficios, aunque se conoce su importancia de 
contar con él, (2) apropiación de la funcionalidad, este nivel de apropiación, implica familiarizarse con el funcionamiento del recurso tecnológico, y (3) apropiación de nuevas formas de aprendizaje, este nivel es el más profundo de apropiación, que involucra usar la tecnología como una herramienta de aprendizaje para desarrollar proyectos, que sean relevantes a condiciones locales, intereses y problemas y vinculados con asignaturas escolares.

La cuestión interesante con este concepto es invitar a la reflexión para analizar el potencial que puede llegar a tener el lograr que los usuarios adopten, adapten y se "adueñen" de la tecnología como parte integral de las actividades cotidianas, valorando su uso y transformándola a necesidades específicas. Se pueden vislumbrar nuevos caminos y potencialidades, se puede ir más allá de su uso y generar nuevos proyectos. Se requiere hacer un análisis de sus potencialidades, de sus retos y dar un salto en el uso de las tecnologías, de tal forma que los usuarios puedan llegar a "apropiarse" de ellas y generar nuevas potencialidades para las mismas.

\section{EL APRENDIZAJE MÓVIL DESDE IMPLEMENTACIONES PRÁCTICAS: EL CASO DEL TECNOLÓGICO DE MONTERREY}

\section{Contexto de la institución}

El Sistema Tecnológico de Monterrey es una institución de educación superior en México, creada en 1943 por la sociedad civil. Es un sistema multicampi (33 campus en 28 ciudades de la República Mexicana), 21 oficinas de Enlace Internacional, siete sedes en Latinoamérica, con presencia en 43 países y redes académicas con más de 500 instituciones en todo el mundo. Cuenta con cuatro salas de transmisión satelital en Campus Monterrey, dos salas transmisoras en Campus Estado de México y salas de videoconferencia en los 33 campus y cuatro canales satelitales y por internet. La plataforma tecnológica Blackboard es usada por 8,448 profesores y por 91,672 alumnos.

Un área muy importante del Tecnológico de Monterrey lo constituye la Universidad Virtual (UV). La UV fue fundada en 1989 como un sistema interactivo de educación a distancia con el fin de ampliar la cobertura docente y llevar educación sin importar las limitaciones geográficas. En sus inicios se centró en el uso de la tecnología satelital para la transmisión de cursos con el apoyo de Internet para la interacción. A partir de 1999 se inicia una evolución con el incremento de cursos ofertados totalmente en Internet. 
En sus ambientes de aprendizaje, el Tecnológico de Monterrey ha evolucionado el salón de clases: desde el sistema presencial (con el que surgió la institución), a la incorporación de tecnología en sus clases para generar ambientes multimodales (cuando decide incorporar el uso de plataformas para complementar los sistemas presenciales), a la educación por satélite con ambientes de blended learning (con el que surgió la universidad virtual), a la educación en línea con ambientes de $e$ learning (cuando se decide dejar las clases satelitales en la universidad virtual e irse con cursos completamente en línea), hasta lo que ahora se está incorporando: aprendizaje móvil con ambientes de m-learning (incorporando recursos para dispositivos móviles).

\section{Antecedentes de las implementaciones de mlearning en la institución}

En las estadísticas de junio del año 2007, sobre el acceso a la tecnología de información, Red de Comunicaciones e Internet en México (Sistema Nacional Estadístico de Información Geográfica, en el Instituto Nacional de Estadística de Geografía e Informática y en el International Communication Union) la institución encontró que había una "ventana" de oportunidad en educación si se incorporaban recursos que pudieran ser accesibles por medio de dispositivos móviles, porque hay una marcada diferencia entre la población mexicana que está suscrita a servicios de Internet (22 millones de habitantes) y quienes están suscritos a telefonía móvil (61,9 millones de habitantes).

En la institución se inició con dos proyectos piloto, por un lado, se incorporó el uso del podcast como un método o canal de entrega de contenidos educativos e instruccionales, en diversos cursos académicos de programas educativos a distancia; y, por el otro, se incorporó el manejo de mensajes de texto vía teléfonos celulares para establecer comunicación entre los alumnos y los contenidos del curso; aunque se pensó que se podía ir más allá de los mensajes de textos en este proyecto, no se pudieron incorporar mensajes multimedia por carecerse de la infraestructura tecnológica y de soporte de telefonía celular con que se contaba en el país en esos momentos (Burgos, 2007).

Como resultado de la implementación de ambos proyectos, en el verano del año 2007 la institución decide hacer una valoración de las tecnologías de telefonía celular disponible en el país, encontrando la posibilidad en sólo un proveedor que permitía estar en la tercera generación (ancho de banda para mensajes de audio y video y sistema de transferencia de entrega de ambos en demanda). Por tal motivo, la institución establece una alianza estratégica con IUSACELL para incluir materiales 
con audio y video en demanda para poder ofrecer a los estudiantes la posibilidad de manejar este tipo de medios de aprendizaje a un costo relativamente bajo.

En sus inicios la institución nombraba el proyecto como "Aprendizaje en movimiento", pero posterior a los pilotajes decidió cambiarlo por "aprendizaje móvil" (mlearning) para que tuviera mayor aceptación con los usuarios. El Tecnológico de Monterrey delimita el aprendizaje móvil como la convergencia del "e-learning" y el uso de la tecnología móvil, donde se integran tres elementos fundamentales de flexibilidad en el tiempo, espacio y lugar; con la intención de fortalecer las capacidades de interacción y apoyo en el proceso de enseñanza-aprendizaje, así como de comunicación en los distintos procesos del modelo educativo.

\section{Implementaciones de mlearning en la institución}

La integración de recursos tecnológicos de aprendizaje móvil en la institución surgió en los ambientes de educación a distancia y se ha propagado en sus ambientes multimodales, con diferentes proyectos. En el momento en que se presenta este artículo, se encuentran trabajando con recursos de mlearning: 36 grupos y 5970 alumnos de seis maestrías usando aprendizaje móvil; 74 grupos y 3286 alumnos de licenciatura; y 20 grupos y 380 alumnos de preparatoria. A continuación se describen tres grandes momentos de implementaciones en la institución:

- Maestría en aprendizaje móvil (septiembre-noviembre 2007): en septiembre del año 2007 la Universidad Virtual del Tecnológico de Monterrey inició su primera maestría con aprendizaje móvil (Maestría en Administración y Tecnologías de Información -MATI-) y ese mismo mes se iniciaron los estudios de impacto (esta información será detallada en el apartado de investigaciones). Fue así como se pasó de ambientes de e-learning con uso de recursos sincrónicos (conferencias magistrales, video por Internet, Radio Chat, Chat) y recursos asincrónicos (videoteca digital, biblioteca digital, videos y audios, multimedios, lecturas y artículos y ligas de Internet) a los ambientes de m-learning con recursos para dispositivos móviles (videos, audios, lecturas, ejercicios interactivos, actividades de colaboración, entre otros) accesibles a través de teléfonos celulares, PDAphones, Smartphones, acceso a Wap, BlackBerry, agendas Digitales (PDA's), Tablet PC, UMPC, reproductores de audio y video digitales portátiles, $e$-Books.

- En el periodo septiembre-noviembre del año 2007 fueron cuatro los cursos impactados con recursos para dispositivos móviles en la Maestría en Tecnologías de Información y Administración (MATI), con 159 alumnos suscritos al servicio. 
- Implementaciones en programas de educación a distancia (enero-diciembre de 2008): En el periodo de enero-marzo de 2008 fueron 14 cursos impactados de MATI, en la Maestría en Administración (MA) y en Programas Empresariales, con 543 alumnos suscritos al servicio y se integró el envío de mensajes SMS por parte de Consejería Académica.

- En el periodo de abril-junio de 2008 fueron 18 los cursos impactados de los programas de MATI, MA, Maestría en Comercio Electrónico (MCE), Maestría en Innovación y Desarrollo Empresarial (MIDE) y en los Programas Empresariales. En ese periodo se inicia también con dos cursos de los programas de la Escuela de Graduados en Educación y a partir de agosto de 2008 se impacta en los programas de esta Escuela: Maestría en Educación y Maestría en Tecnología Educativa.

- La plataforma en la que se apoyan los cursos es Blackboard, a partir de la implementación de esta modalidad el estudiante encuentra nuevos gráficos e imágenes que le indican en qué formato se encuentran los recursos tecnológicos de sus actividades. Estas imágenes representan la forma en que puede disponer de ese recurso, por ejemplo: una iPod si es un recurso de audio o video que puede bajar en ese dispositivo, un celular si es un recurso del que puede disponer para telefonía móvil, una imagen de un $P D F$ si es un recurso de texto o una imagen con cámara y sonido si es un recurso multimedia que puede acceder desde la plataforma.

- Implementaciones de recursos de mlearning en programas presenciales con ambientes multimodales (julio-diciembre de 2008): a partir de julio de 2008 la incorporación de recursos tecnológicos con dispositivos móviles trascendió los ambientes de educación a distancia de la institución y se incorpora en los ambientes multimodales, en el nivel educativo de bachillerato, en varios campus de la institución y en el nivel educativo de profesional, en los Campus Santa Fe y Ciudad de México. En estos dos campus se otorgaron Blackberrys a los más de 3000 alumnos de nuevo ingreso, para integrarlos en los ambientes de aprendizaje de las diversas carreras profesionales, a partir de agosto de 2008. En ambos niveles educativos los directivos y profesores recibieron capacitación para diseñar y producir recursos para aprendizaje móvil.

Otro tipo de proyecto de incorporación de mlearning en los ambientes de nivel profesional fue el que se dio a partir de agosto 2008 en los campus Estado de México, Querétaro y Toluca, donde se convocó a la comunidad estudiantil y 
académica a concursar, en equipos, con proyectos que desarrollaran aplicaciones para los dispositivos móviles y recursos para estos dispositivos. A los equipos que presentaron sus proyectos se les apoyó con Blackberrys para sus trabajos.

Dos campus adicionales que han recibido capacitación en esta temática han sido el campus Monterrey (profesores de profesional de la Dirección de Finanzas y Administración) y el campus San Luis Potosí (profesores de diversas carreras profesionales), a través de un diplomado en innovación educativa en ambientes de aprendizaje basados en tecnología, impartido por la Cátedra en Investigación en Innovación de Tecnología y Educación (http://www.ruv.itesm.mx/convenio/ catedra/homedoc.htm) y por el Centro para la Innovación en Tecnología y Educación (Innov@te http://www.itesm.mx/innovate).

\section{Recursos tecnológicos y su integración en los ambientes de aprendizaje}

En el diseño y producción de los recursos tecnológicos para dispositivos móviles han participado directivos de programa, directivos del centro de innovación de la institución, profesores y equipo multidisciplinar del área de tecnología educativa (diseñadores instruccionales, diseñadores gráficos, informáticos y programadores Web). Ramírez (2009) menciona que, en la apuesta que hace la institución para incorporar estos recursos tecnológicos en los ambientes de aprendizaje, se confía en las ventajas de proporcionar mayor flexibilidad para el acceso de contenidos educativos, personalización de experiencias de aprendizaje, desarrollo y fortalecimiento de habilidades profesionales y mayor efectividad del aprendizaje por el tiempo de atención.

Son múltiples las actividades que se han incorporado en los ambientes de aprendizaje a través de los dispositivos móviles: acceso a Servicios al Usuario; casos; cápsulas de reforzamiento de clases; ejercicios; simulaciones; ejemplificaciones; coevaluaciones y autoevaluaciones, consulta de calificaciones, mensajes, calendarios, consultas de equipos de trabajo, recursos de audio y video (podcast), radio chat móvil, canal en vivo, recursos de audio y video.

Estas actividades se dan a través del soporte de tres servicios: podcasting, tecnología Really Simple Syndication (RSS) y telefonía móvil. La tecnología podcasting se refiere a la descarga de archivos educativos visuales o auditivos entregados por medio de una suscripción gratis a través de una red (RSSNews Reader). Una vez que el usuario se suscribe, los archivos son distribuidos regularmente y pueden ser accedidos con un dispositivo móvil o computadora personal en cualquier 
lugar, espacio y momento. El servicio RSS también se puede utilizar para conocer noticias y contenidos de diferentes sitios web en un mismo canal, dando la facilidad al usuario de no tener que navegar por diferentes páginas para conocer noticias de su interés a los que se está suscrito. El servicio de mensajería de texto corto o short message service (SMS de sus siglas en inglés) de la telefonía móvil consiste en enviar alertas a los celulares de los alumnos de carácter académico y de asesoría. Para que un estudiante tenga acceso a este recurso debe suscribirse (sin ningún costo). En la actualidad en este servicio sólo se cuenta con envío de mensajes tipo texto.

El diseño de actividades en un ambiente de aprendizaje se encuentra muy vinculado con las concepciones pedagógicas para el diseño. Mientras que en el $e$ learning se encuentran más actividades de lecturas, texto y gráficas para describir las instrucciones, en el m-learning se usa más la voz, los gráficos y las animaciones para las instrucciones y se promueve el aprendizaje de campo. Pero, es conveniente señalar, que el diseño de actividades no se encuentra sólo en la forma de "entrega de las actividades" (si se usa voz en lugar de texto, por ejemplo), sino en el aprendizaje que quiere lograrse, el contenido que va a transmitirse y la estrategia que va a utilizarse.

\section{Investigaciones a partir de la implementación de m-learning en la institución}

Desde la incorporación de los ambientes de aprendizaje móviles en los programas de la institución, se iniciaron los estudios de las implementaciones de estos recursos, a través del Centro para la Innovación en Tecnología y Educación (Innov@TE) y del grupo de investigadores de la Cátedra de Investigación de Innovación en Tecnología y Educación (pagina Web http://www.tecvirtual.itesm.mx/convenio/ catedra/homedoc.htm). A continuación se describen los diversos estudios con sus objetivos y resultados, que se han desarrollado desde el verano 2007 hasta mayo 2008. Finalmente se presenta un proyecto a gran escala que se está generando de agosto 2008 a junio 2009.

- Pilotaje (Burgos, 2007). El objetivo fue explorar el uso de podcast como canal de entrega y envío de mensajes de texto para comunicación con alumnos de avisos y contenido de cursos. Los resultados fueron: (1) Se mandaron mensajes de texto a celulares de los estudiantes a través de servicio de entrega (no se contaba con portal móvil) y, (2) Se establece una alianza estratégica con IUSACELL para incluir materiales con audio y video en demanda. 
- Dispositivos de mobile learning para ambientes virtuales: Investigación de implicaciones en el diseño y la enseñanza (Ramírez, 2008a). El objetivo fue analizar las implicaciones en las prácticas de diseño y la enseñanza cuando se incorporan dispositivos móviles en los ambientes de aprendizaje virtuales. Los resultados fueron: (1) implicaciones organizacionales (costo y cambio organizacional con visión, planeación y compromiso), (2) implicaciones estructurales e infraestructura (trabajo multidisciplinar), (3) implicaciones sociales (capital social), (4) implicaciones de análisis previo (perfil tecnológico y anticipación en diseño), (5) implicaciones tecnológicas (entrega y características de contenido) y, (6) implicaciones de pensamiento y de acción (creatividad y vinculación con el mundo real).

- Competencias aplicadas por alumnos de posgrado para el uso de dispositivos m-learning (Herrera, Lozano y Ramírez, 2008). El objetivo fue analizar los conocimientos, habilidades y actitudes que están aplicando los alumnos en el uso de los dispositivos m-learning en dos cursos de posgrado impartidos en la modalidad de educación a distancia. Los resultados fueron: (1) la integración al currículo de los materiales y dispositivos para m-learning, permiten al alumno desarrollar ciertas habilidades (uso computacional, comunicación, liderazgo, autoaprendizaje y productividad); (2) también se encontró que los alumnos no tenían desarrolladas las competencias de manejo de herramientas computacionales para sincronizar los recursos alos celulares yel iPod. Seidentificó la necesidad de desarrollar habilidades de autodirección (autoadministración, evaluación y selección de información, creatividad, comunicación y trabajo colaborativo).

- Competencias que requieren los alumnos de MATI para aprender con dispositivos m-learning (Lozano, 2007). El objetivo fue determinar y definir las competencias clave requeridas por los estudiantes para adquirir adecuadamente conocimientos a través de dispositivos $m$-learning a través de estudiantes del curso de Estrategias del Pensamiento Crítico para la Investigación Educativa. Los resultados fueron: las competencias requeridas por estudiantes son: capacidad de adaptación al ambiente m-learning; resolución de problemas educativos mediante soluciones creativas; habilidad en el uso de los dispositivos electrónicos móviles de audio y video; capacidad de intercambio de información entre los dispositivos y habilidad para acceder a los archivos descargables (podcast).

- M-learning como herramienta para el desarrollo de habilidades de debate en foros asincrónicos de comunicación (Marcos, Tamez y Lozano, 2008). El 
objetivo fue verificar si existe alguna diferencia significativa entre las habilidades de debate de los alumnos que utilizaron la herramienta de $m$-learning por medio de dispositivos móviles en comparación con los que no la usaron y proponer herramientas alternativas para incrementar el aprovechamiento académico. Los resultados fueron: (1) la sensibilidad del post test: esto es una curva muy sesgada a la derecha. No hay una normalidad, (2) por la forma en que se evaluó la rúbrica los puntajes fueron muy altos, (3) algunos de los podcast eran en inglés (las capacidades lingüísticas pudieron influir) y (4) el tiempo de intervención fue muy pequeño, esto tiene que ver con cuántas veces los alumnos escuchan los podcast o leen los textos; (5) Los textos pueden ser más manipulables que los audios en términos de estrategias de aprendizaje y eso pudo influir en los resultados y, (6) En una encuesta adicional realizada a los estudiantes, mencionaron que el empleo del m-learning les permitía mejorar su manejo personal del tiempo y que los motivaba más que simplemente la plataforma de Blackboard.

Actualmente (de agosto 2008 a junio 2009) se está realizando un Proyecto a gran escala de m-learning con siete sub-proyectos, a través de la Cátedra de Innovación en Tecnología y Educación.

El objetivo del proyecto es generar conocimiento del balance de los componentes pedagógicos, tecnológicos y de contenido, a partir de la aplicación de dispositivos móviles en ambientes educativos, a través de estudios con alumnos, profesores, administradores y personal de tecnología educativa, que permitan la toma de decisiones fundamentadas. La pregunta de investigación del proyecto a gran escala es ¿́cómo balancear los componentes pedagógicos, tecnológicos y de contenido para la adquisición y desarrollo de competencias a través de m-learning?

A partir de esta gran pregunta del proyecto, se derivan siete subproyectos que exploran diversas vertientes de las aplicaciones. En la tabla 1 se delinean los siete subproyectos. 
Recursos Tecnológicos Para El Aprendizaje Móvil (Mlearning) y su Relación con los Ambientes...

\begin{tabular}{|c|c|c|c|c|}
\hline $\begin{array}{c}\text { Equipo de } \\
\text { investigadores }\end{array}$ & Subproyecto & $\begin{array}{l}\text { Pregunta de } \\
\text { investigación }\end{array}$ & Objetivo & Metodología \\
\hline $\begin{array}{l}\text { Ana Isabel } \\
\text { Ramos, } \\
\text { Alberto Herrera } \\
\text { y María Soledad } \\
\text { Ramírez }\end{array}$ & $\begin{array}{l}\text { Desarrollo de } \\
\text { habilidades } \\
\text { cognitivas con el } \\
\text { uso de recursos } \\
\text { de m-learning }\end{array}$ & $\begin{array}{l}\text { ¿Cuáles habilidades } \\
\text { cognitivas se están } \\
\text { desarrollando en los } \\
\text { estudiantes a través } \\
\text { de los recursos } \\
\text { de m-learning que } \\
\text { son integrados en } \\
\text { los ambientes de } \\
\text { aprendizaje? }\end{array}$ & $\begin{array}{l}\text { Analizar el } \\
\text { contenido de } \\
\text { los recursos de } \\
\text { m-learning en } \\
\text { cuatro cursos de } \\
\text { nivel profesional, } \\
\text { con el fin de } \\
\text { identificar cómo se } \\
\text { trata de promover } \\
\text { el desarrollo } \\
\text { de habilidades } \\
\text { cognitivas en el } \\
\text { alumno. }\end{array}$ & \begin{tabular}{|l} 
Metodología de casos múltiples que \\
empleó instrumentos mixtos, tanto \\
cuantitativos como cualitativos: \\
- $\quad$ Entrevistas semi estructuradas \\
a profesores, desarrolladores y \\
directivos. \\
- Cuestionarios auto dirigidos \\
para los alumnos, profesores y \\
desarrolladores. \\
Focus group con los \\
desarrolladores de recursos, \\
profesores y estudiantes. \\
Análisis de documentos \\
significativos y observación \\
(mediciones no intrusivas a los \\
recursos a través del repositorio, \\
documentos del proyecto de \\
formación de mlearning). \\
Aplicación en campus Santa Fe y Cd. \\
de México.
\end{tabular} \\
\hline $\begin{array}{l}\text { Josefina } \\
\text { Contreras, Alberto } \\
\text { Herrera y María } \\
\text { Soledad Ramírez }\end{array}$ & \begin{tabular}{|l} 
Componentes \\
pedagógicos, \\
tecnológicos y \\
de diseño en \\
la producción \\
de materiales \\
para dispositivos \\
móviles
\end{tabular} & $\begin{array}{l}\text { ¿Cuáles son } \\
\text { los elementos } \\
\text { pedagógicos, } \\
\text { tecnológicos y de } \\
\text { diseño que deben } \\
\text { ser considerados } \\
\text { para la producción } \\
\text { de materiales } \\
\text { adecuados para } \\
\text { dispositivos } \\
\text { móviles? }\end{array}$ & $\begin{array}{l}\text { Analizar el proceso } \\
\text { de producción } \\
\text { de recursos } \\
\text { para dispositivos } \\
\text { m-learning en } \\
\text { dos campus de } \\
\text { una institución } \\
\text { educativa } \\
\text { mexicana con el } \\
\text { fin de identificar } \\
\text { los elementos } \\
\text { pedagógicos, } \\
\text { tecnológicos y } \\
\text { de diseño que } \\
\text { los componen } \\
\text { y poder aportar } \\
\text { conocimientos del } \\
\text { tema a la }\end{array}$ & $\begin{array}{l}\text { Metodología de casos múltiples que } \\
\text { empleó instrumentos mixtos, tanto } \\
\text { cuantitativos como cualitativos, } \\
\text { para identificar la producción desde } \\
\text { diversas áreas disciplinares (ciencias, } \\
\text { humanidades, entre otras): } \\
\text { - } \quad \text { Entrevistas semi estructuradas } \\
\text { a profesores, desarrolladores y } \\
\text { directivos. } \\
\text { Cuestionarios auto dirigidos } \\
\text { para los alumnos, profesores y } \\
\text { desarrolladores. } \\
\text { Focus group con los } \\
\text { desarrolladores de recursos, } \\
\text { profesores y estudiantes. }\end{array}$ \\
\hline
\end{tabular}


Recursos Tecnológicos Para El Aprendizaje Móvil (Mlearning) y su Relación con los Ambientes...

\begin{tabular}{|c|c|c|c|c|}
\hline $\begin{array}{c}\text { Equipo de } \\
\text { investigadores }\end{array}$ & Subproyecto & $\begin{array}{l}\text { Pregunta de } \\
\text { investigación }\end{array}$ & Objetivo & Metodología \\
\hline & & & $\begin{array}{l}\text { comunidad } \\
\text { educativa y a los } \\
\text { desarrolladores de } \\
\text { software, hardware } \\
\text { y recursos de } \\
\text { tecnología móvil. }\end{array}$ & $\begin{array}{l}\text { - Análisis de documentos } \\
\text { significativos y observación } \\
\text { (mediciones no intrusivas a los } \\
\text { recursos a través del repositorio, } \\
\text { documentos del proyecto de } \\
\text { formación de m-learning y } \\
\text { documentación institucional del } \\
\text { proyecto). } \\
\text { Aplicación en campus Santa Fe y Cd. } \\
\text { de México. }\end{array}$ \\
\hline $\begin{array}{l}\text { Ma. Soledad } \\
\text { Moctezuma, } \\
\text { Alberto Herrera } \\
\text { y María Soledad } \\
\text { Ramírez }\end{array}$ & $\begin{array}{l}\text { Características } \\
\text { predominantes } \\
\text { en la generación } \\
\text { Net que favorecen } \\
\text { el aprendizaje al } \\
\text { usar dispositivos } \\
\text { móviles }\end{array}$ & $\begin{array}{l}\text { ¿Qué características } \\
\text { predominantes } \\
\text { en la generación } \\
\text { Net pueden ser } \\
\text { explotadas para } \\
\text { favorecer el } \\
\text { aprendizaje al } \\
\text { usar dispositivos } \\
\text { móviles? }\end{array}$ & \begin{tabular}{|l} 
Analizar las \\
características \\
relacionadas con \\
la generación Net \\
en estudiantes \\
de bachillerato \\
y licenciatura \\
con el fin de \\
identificar cuáles \\
características \\
pueden ser \\
aprovechadas \\
para promover \\
aprendizajes \\
a través de \\
dispositivos \\
móviles.
\end{tabular} & $\begin{array}{l}\text { Estudio mixto, descriptivo y } \\
\text { correlacional, con dos fases, una } \\
\text { primera fase fue descriptiva de las } \\
\text { características de Generación Net y en } \\
\text { una segunda fase, se correlacionaron } \\
\text { las características de los estudiantes } \\
\text { con los aprendizajes promovidos } \\
\text { a través de los recursos. Los } \\
\text { instrumentos que se aplicaron fueron: } \\
\text { Entrevistas semi estructuradas } \\
\text { a profesores, desarrolladores y } \\
\text { directivos. } \\
\text { Cuestionarios auto dirigidos } \\
\text { para los alumnos, profesores y } \\
\text { desarrolladores. } \\
\text { Focus group con los } \\
\text { desarrolladores de recursos, } \\
\text { profesores y estudiantes. } \\
\text { Análisis de documentos } \\
\text { significativos y observación } \\
\text { (mediciones no intrusivas a los } \\
\text { recursos a través del repositorio, } \\
\text { documentos del proyecto de } \\
\text { formación de mlearning y } \\
\text { documentación institucional del } \\
\text { proyecto). } \\
\text { Aplicación en campus Santa Fe y Cd. } \\
\text { de México. }\end{array}$ \\
\hline
\end{tabular}




\begin{tabular}{|c|c|c|c|c|}
\hline $\begin{array}{c}\text { Equipo de } \\
\text { investigadores }\end{array}$ & Subproyecto & $\begin{array}{l}\text { Pregunta de } \\
\text { investigación }\end{array}$ & Objetivo & Metodología \\
\hline $\begin{array}{l}\text { Carlos Gallardo, } \\
\text { Dulce Rivera y } \\
\text { María Soledad } \\
\text { Ramírez }\end{array}$ & \begin{tabular}{|l} 
Análisis de la \\
implementación \\
académica de \\
los recursos de \\
m-learning y su \\
impacto en el \\
desarrollo de \\
competencias \\
profesionales \\
en estudiantes \\
universitarios.
\end{tabular} & $\begin{array}{l}\text { ¿Cómo la } \\
\text { implementación del } \\
\text { m-learning en el } \\
\text { ambiente académico } \\
\text { puede favorecer } \\
\text { al desarrollo de } \\
\text { competencias } \\
\text { profesionales en los } \\
\text { alumnos? }\end{array}$ & $\begin{array}{l}\text { Analizar la } \\
\text { implementación } \\
\text { académica de } \\
\text { recursos de } \\
\text { m-learning en } \\
\text { los procesos } \\
\text { educativos de } \\
\text { estudiantes de } \\
\text { nuevo ingreso } \\
\text { de tres carreras } \\
\text { profesionales en } \\
\text { una Universidad } \\
\text { privada mexicana } \\
\text { multicampus, } \\
\text { para identificar } \\
\text { cuáles son las } \\
\text { competencias } \\
\text { profesionales que } \\
\text { dichos recursos } \\
\text { desarrollan los } \\
\text { estudiantes de } \\
\text { nivel universitario. }\end{array}$ & $\begin{array}{l}\text { Investigación cualitativa con el método } \\
\text { de estudio de casos múltiples. Los } \\
\text { instrumentos que se aplicaron fueron: } \\
\text { - } \quad \text { Entrevistas semi estructuradas a } \\
\text { coordinadores. } \\
\text { - Cuestionarios auto dirigidos para } \\
\text { los alumnos. } \\
\text { - } \quad \text { Aplicación en campus Santa Fe. }\end{array}$ \\
\hline $\begin{array}{l}\text { Eliseo Arturo } \\
\text { Sandoval, } \\
\text { Rosa García y } \\
\text { María Soledad } \\
\text { Ramírez }\end{array}$ & $\begin{array}{l}\text { Competencias } \\
\text { tecnológicas y los } \\
\text { conocimientos en } \\
\text { la capacitación } \\
\text { para la producción } \\
\text { de recursos } \\
\text { tecnológicos } \\
\text { de dispositivos } \\
\text { móviles de tercera } \\
\text { generación. }\end{array}$ & $\begin{array}{l}\text { ¿Cuáles son las } \\
\text { competencias } \\
\text { tecnológicas } \\
\text { y los } \\
\text { conocimientos } \\
\text { que deben ser } \\
\text { considerados } \\
\text { para la } \\
\text { capacitación en } \\
\text { la producción } \\
\text { de recursos } \\
\text { tecnológicos } \\
\text { de dispositivos } \\
\text { móviles } \\
\text { de tercera } \\
\text { generación? } \\
\end{array}$ & $\begin{array}{l}\text { Analizar las } \\
\text { competencias } \\
\text { tecnológicas } \\
\text { y los } \\
\text { conocimientos } \\
\text { que tienen los } \\
\text { facilitadores } \\
\text { cuando } \\
\text { capacitan en } \\
\text { la producción } \\
\text { de recursos } \\
\text { tecnológicos } \\
\text { de } 3 G \text { para } \\
\text { dispositivos } \\
\text { móviles. }\end{array}$ & $\begin{array}{l}\text { Metodología de casos múltiples } \\
\text { que empleó instrumentos } \\
\text { mixtos, tanto cuantitativos como } \\
\text { cualitativos: } \\
\text { - Entrevistas semi estructuradas } \\
\text { a profesores, desarrolladores, } \\
\text { facilitadores de capacitación y } \\
\text { directivos. } \\
\text { - Cuestionarios auto } \\
\text { dirigidos para profesores, } \\
\text { facilitadores de capacitación y } \\
\text { desarrolladores. } \\
\text { - Focus group con los } \\
\text { desarrolladores de recursos, } \\
\text { facilitadores de capacitación y } \\
\text { profesores. }\end{array}$ \\
\hline
\end{tabular}


Recursos Tecnológicos Para El Aprendizaje Móvil (Mlearning) y su Relación con los Ambientes...

\begin{tabular}{|c|c|c|c|c|}
\hline $\begin{array}{c}\text { Equipo de } \\
\text { investigadores }\end{array}$ & Subproyecto & $\begin{array}{l}\text { Pregunta de } \\
\text { investigación }\end{array}$ & Objetivo & Metodología \\
\hline & & & & $\begin{array}{l}\text { - Observaciones de prácticas de } \\
\text { capacitación (videograbación de } \\
\text { las sesiones para analizarlas). } \\
\text { - Análisis de documentos } \\
\text { significativos y observación } \\
\text { (mediciones no intrusivas a los } \\
\text { recursos a través del repositorio, } \\
\text { documentos del proyecto de } \\
\text { formación de m-learning y } \\
\text { documentación institucional del } \\
\text { proyecto). } \\
\text { Aplicación en campus Estado de } \\
\text { México, Santa Fe y Monterrey. }\end{array}$ \\
\hline $\begin{array}{l}\text { Dagoberto } \\
\text { Gerardo Pérez, } \\
\text { Rosa García y } \\
\text { María Soledad } \\
\text { Ramírez }\end{array}$ & $\begin{array}{l}\text { Interacciones } \\
\text { educativas y } \\
\text { sociales en } \\
\text { m-learning y en } \\
\text { e-learning }\end{array}$ & $\begin{array}{l}\text { ¿Qué } \\
\text { interacciones } \\
\text { educativas y } \\
\text { sociales son } \\
\text { necesarias para } \\
\text { mejorar el m- } \\
\text { learning a partir } \\
\text { del e-learning? }\end{array}$ & $\begin{array}{l}\text { Analizar las } \\
\text { semejanzas } \\
\text { y diferencias } \\
\text { entre el diseño } \\
\text { instruccional, } \\
\text { las } \\
\text { interacciones } \\
\text { educativas y las } \\
\text { interacciones } \\
\text { sociales en } \\
\text { ambientes de } \\
\text { e-learning y } \\
\text { de m-learning } \\
\text { en dos cursos } \\
\text { de posgrado } \\
\text { que combinan } \\
\text { ambos } \\
\text { ambientes. }\end{array}$ & $\begin{array}{l}\text { Metodología de casos múltiples que } \\
\text { empleó instrumentos mixtos, tanto } \\
\text { cuantitativos como cualitativos: } \\
\text { - Entrevistas semi estructuradas } \\
\text { a coordinadores, profesores } \\
\text { titulares y tutores y diseñadores } \\
\text { instruccionales de dos cursos } \\
\text { (psicología del aprendizaje y } \\
\text { fundamentos de la investigación). } \\
\text { - Cuestionarios auto dirigidos para } \\
\text { estudiantes. } \\
\text { - Focus group con los estudiantes. } \\
\text { - Observaciones de foros de } \\
\text { discusión (análisis del discurso } \\
\text { en Blackboard). } \\
\text { - Análisis de documentos } \\
\text { significativos y observación } \\
\text { (mediciones no intrusivas a } \\
\text { los recursos a través de la } \\
\text { plataforma Blackboard, diseño } \\
\text { de los cursos e integración de } \\
\text { recursos m-learning en el diseño, } \\
\text { documentos de los cursos y } \\
\text { documentación institucional del } \\
\text { proyecto). }\end{array}$ \\
\hline
\end{tabular}


Recursos Tecnológicos Para El Aprendizaje Móvil (Mlearning) y su Relación con los Ambientes...

\begin{tabular}{|c|c|c|c|c|}
\hline $\begin{array}{c}\text { Equipo de } \\
\text { investigadores }\end{array}$ & Subproyecto & $\begin{array}{l}\text { Pregunta de } \\
\text { investigación }\end{array}$ & Objetivo & Metodología \\
\hline & & & & $\begin{array}{l}\text { Aplicación en dos cursos de } \\
\text { la Escuela de Graduados } \\
\text { en Educación (análisis de } \\
\text { estudiantes de diferentes } \\
\text { campus). }\end{array}$ \\
\hline $\begin{array}{l}\text { José de Jesús } \\
\text { Rodríguez, } \\
\text { Dulce Camacho } \\
\text { y Yolanda } \\
\text { Cázares }\end{array}$ & $\begin{array}{l}\text { Habilidades de } \\
\text { autodirección } \\
\text { presentes al } \\
\text { trabajar con } \\
\text { dispositivos } \\
\text { móviles (m- } \\
\text { learning) }\end{array}$ & $\begin{array}{l}\text { ¿Qué } \\
\text { habilidades de } \\
\text { autodirección } \\
\text { son utilizadas a } \\
\text { través del uso } \\
\text { de dispositivos } \\
\text { móviles? }\end{array}$ & $\begin{array}{l}\text { Identificar } \\
\text { aquéllos } \\
\text { componentes } \\
\text { de auto- } \\
\text { dirección que } \\
\text { son utilizados } \\
\text { por usuarios } \\
\text { de dispositivos } \\
\text { móviles. }\end{array}$ & $\begin{array}{l}\text { Metodología cuantitativa, con } \\
\text { encuestas a los alumnos. } \\
\text { Aplicación en campus Santa Fe, } \\
\text { Cd. de México y Toluca. }\end{array}$ \\
\hline
\end{tabular}

Tabla 1. Siete estudios de impacto sobre las incorporaciones de m-learning en ambientes de aprendizaje

El objetivo que se tiene al realizar estas investigaciones es llegar a generar conocimiento práctico sobre los resultados de las implementaciones de los recursos tecnológicos a través de dispositivos móviles que pueda ser útil en tres niveles: a) por un lado, proporcionar información a la institución donde se están realizando estas innovaciones para que pueda tomar decisiones fundamentadas, con los resultados y la identificación de los aspectos críticos que se suscitan en las implementaciones; b) por otro lado, brindar datos prácticos a la comunidad educativa, en general, que se encuentre interesada en conocer qué sucede con la implementación de estos recursos en los ambientes de aprendizaje y; c) finalmente, proporcionar resultados empíricos para contribuir en la generación de conocimiento en el área de la tecnología educativa y la educación a distancia, con innovaciones educativas basadas en evidencias.

\section{ALGUNAS REFLEXIONES Y RETOS EN LA IMPLEMENTACIÓN DE MLEARNING}

La apuesta por incorporar innovaciones tecnológicas en los ambientes de aprendizaje no es un proceso fácil. En estas apuestas intervienen muchos elementos de gran peso: finalidades, sentidos, creencias, supuestos, cambios, metas, búsquedas de mejoras... Intervienen también muchas consideraciones: costos de recursos humanos, costos de infraestructura, costos de tiempos, espacios... Interviene 
también el considerar la forma de implementación: ¿por dónde se inicia?, ¿con qué se continuaría?, ¿a quiénes se involucra primero?, ¿a quiénes después?, ¿cómo se les integra?... Lo cierto es que el incorporar innovaciones tecnológicas en ambientes educativos trae consigo mucha planeación detrás, mucho trabajo en la implementación y mucho esfuerzo para conocer sus resultados. Pero también quiero decir que, en cada una de esas etapas, se encuentran los retos interesantes del trabajo académico.

Desde una mirada externa (que me resulta difícil por ser, en cierto sentido, juez y parte de estas implementaciones) puedo visualizar un proceso realmente muy rápido y muy interesante. Es un proceso tan rápido que se dificulta captar lo que está pasando con cada uno de los agentes intervinientes y muy interesante porque también se puede ver el ánimo de la "novedad", y la contraparte en el escepticismo de cuánto pueden llegar a aportar estos recursos que tanto trabajo está resultando para quienes los generan. Y en este panorama están también los claroscuros, las reflexiones del querer creer en sus potencialidades, pero a la vez dudar de su efectividad, o viceversa.

En este panorama, de mucha incertidumbre aún y de algunas certezas, se planteó el objetivo de este artículo, que se centró en tratar de describir la experiencia de la implementación de recursos tecnológicos para el aprendizaje móvil en ambientes educativos multimodales y de educación a distancia, con el fin de analizar sus implicaciones operativas y los retos que expone este tipo de innovaciones educativas.

Sin pretender ser exhaustiva en los análisis me concretaré a analizar tres implicaciones operativas que detectó y tres retos que visualizo en la integración de recursos de aprendizaje móvil.

Por una parte encuentro implicaciones de orden tecnológico donde se requiere de equipos de digitalización y discos duros de capacidad extensa. Los recursos que se estaban grabando para los ambientes multimodales y de educación a distancia se hacían en formato análogo (cinta, cassets), pero, la introducción de recursos de tercera generación requiere que puedan integrarse en dispositivos de celulares, iPods, reproductores de audio y video y estos recursos deben desarrollarse en un formato digital, de tal forma que se necesitan discos duros de capacidad extensa y tecnologías de grabación para recursos digitales. Los productores de estos recursos han podido realizar la tarea, pero, entre los procesos de grabación, edición, catalogación, digitalización y "subirlo" al servidor, los costos de tiempo y esfuerzo han sido realmente altos. 
Otra implicación operativa es la del diseño de la configuración tecnológica, donde se requiere un tratamiento diferente de los que se venía elaborando desde el área de la tecnología educativa, para diseñar recursos que ahora son entregados en "pequeños" dispositivos. Se requiere un tratamiento especial de diseño gráfico porque ha de considerarse que la pantalla de un celular (por ejemplo), dista mucho de una pantalla de computadora. Se requiere también una especial atención a la configuración técnica para que puedan ser reproducidos de manera eficiente en los dispositivos. Estas implicaciones han traído una operación diferente para los diseñadores gráficos, diseñadores instruccionales y programadores que apoyan la generación de los recursos, así como para los mismos profesores. También se ha requerido elaborar formatos especiales para apoyar el diseño (colores, imágenes de asociación, tamaños de letras, tiempos especiales, pesos) y para dar las indicaciones de cómo accesarlos.

La tercera implicación a la que quiero hacer mención va directamente relacionada con el objetivo de este artículo y es la referida a la integración en los ambientes de aprendizaje, sobre todo, en los ambientes de aprendizaje a distancia. En los ambientes de e-learning suelen utilizarse las lecturas digitalizadas, laboratorios remotos, bibliotecas digitales, casos, problemas, ejercicios, objetos de aprendizaje, etc. En mlearning los materiales deben superar no sólo la limitación del tamaño en que lo reproduce el dispositivo y el tamaño de la pantalla, como lo acabo de mencionar, sino que más allá de las limitaciones de "entrega" se encuentra la implicación de la relevancia de los recursos a utilizar para dar un "valor agregado" al ambiente de aprendizaje. Algunos materiales de m-learning que están siendo integrados son vídeos, cápsulas, mapas conceptuales, gráficos, fotografías, audios, objetos de aprendizaje, test, escenarios, casos, conferencias, construcción de soporte al desempeño, simulaciones, etc. Desde este punto conviene preguntarnos cंqué tipo de contenidos son aptos para trabajarse con dispositivos móviles?, ¿qué materiales son óptimos para estos dispositivos? y ¿cómo estos recursos mejoran los aprendizajes en la educación a distancia? Estudiar este punto lo considero de una importancia capital para el futuro de los recursos de aprendizaje móvil.

Ahora bien, a la luz de los resultados que hasta ahora nos han dado las investigaciones, la lista de las implicaciones puede resultar también muy extensa, pero quiero pasar ahora a los retos que, en cierta forma, van muy asociados a las implicaciones.

Un primer reto al que quiero hacer mención va relacionado con lo que mencioné en el marco conceptual de este escrito: las redes inalámbricas. El aprendizaje móvil está sujeto a la infraestructura de redes inalámbricas de los proveedores de los dispositivos móviles, por lo que las fallas externas a las instituciones que proveen 
Recursos Tecnológicos Para El Aprendizaje Móvil (Mlearning) y su Relación con los Ambientes...

los ambientes de aprendizaje pueden representar dificultades y este es un punto a considerar.

Un segundo reto se encuentra en la capacidad de almacenamiento que tienen los dispositivos móviles. En la experiencia descrita, una vez superada la identificación de dispositivos que tuvieran la tecnología para tercera generación, se han suscitado otras cuestiones, como la capacidad de almacenamiento de los recursos y aunque cada día somos testigos de que la tecnología va aumentando en su potencia, aún ahora la capacidad de almacenaje sigue siendo una asignatura pendiente y sigue representando un reto para los programadores e informáticos.

El tercer reto lo veo como punto clave para el aprendizaje móvil: la configuración de contenido de los recursos. Si bien ya lo mencioné como una implicación, quiero retomarlo como un reto, como el mayor reto que encuentro en estos nuevos recursos, porque, más allá de los retos tecnológicos (que tienen una importancia relevante) está la certeza de que los avances científicos van a permitir superarlos en espacios temporales realmente cortos, pero lo que concierne a las prácticas pedagógicas, el diseño de contenidos de los recursos requieren no sólo ciencia y técnica, sino también intención, conocimiento y creatividad para visualizar esos contenidos de una forma diferente a lo que estamos habituados a hacer y a generar. Los contenidos en un ambiente virtual constituyen el eje central de los aprendizajes que buscan promoverse en una situación educativa. Mientras que en el e-learning la organización de los contenidos puede hacerse de múltiples formas: proyectos, unidades, temáticas, definiciones. En m-learning se promueve más la organización atomizada de contenidos, parecido a cuando se trabaja con objetos de aprendizaje (Ramírez, 2008b) cuando se recomienda seccionar los temas en unidades pequeñas de contenido, con información completa y autocontenible, si esto es así cंqué puede "colocarse" en ese recurso que de verdad "desequilibre" el pensamiento e invite a aprendizajes de alto nivel?

Cierro el escrito con una reflexión integradora, el uso de recursos tecnológicos para el aprendizaje móvil ha representado para la institución una apuesta a la innovación, un esfuerzo por aportar nuevos recursos a sus ambientes de aprendizaje, un cambio de paradigmas en la construcción de los materiales para la comunidad académica, porque ha requerido una forma diferente de generar los recursos, una forma diferente en sus diseños gráficos y en sus procesos de integración. Se ha requerido de muchos esfuerzos, hay ya algunos aprendizajes, pero tengo la certeza de que esta actividad apenas está iniciando, lo mejor está por venir, hay un mundo aún por descubrir en el campo del mlearning y tengo la esperanza de que ese campo va a ser realmente gratificante para todos los que estamos involucrados en estos ambientes de educación a distancia. 


\section{REFERENCIAS BIBLIOGRÁFICAS}

Berners-Lee, T. (1999). Weaving the Web: The post Present and Future of the World Wide Web by its inventor. London, Orion: Bussiness Books.

Burgos, V. (2007). Contextualizando el aprendizaje en movimiento. Videoconferencia de trabajo de investigación grabada el 18 de septiembre del 2007; organizada por los integrantes de la Cátedra de Investigación "Innovación en tecnología y educación": Herrera, A.; González, G., Lozano, F. G.; Ramírez, M. S. Recurso inédito para efectos de investigación.

Cátedra de Investigación de Innovación en Tecnología y Educación (2008). Página Web de la Cátedra. [en línea] Disponible en: http://www.ruv.itesm.mx/convenio/ catedra/homedoc.htm [consulta 2009, 2 de Julio]

Clyde, L. (2004). M-learning. Teacher Librarian, 32, 1, (45-46). [en línea] Disponible en: http://o-proquest.umi. com.millenium.itesm.mx:80/pqdlink?di

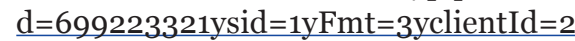
$3693 \mathrm{yRQT}=309 \mathrm{yVName}=\mathrm{PQD}$ [consulta 2007, 17 de Julio]

De Pablos, J. (1998). Nuevas tecnologías aplicadas a la educación: una vía para la innovación. En: De Pablos, J.; Jimenez, J. (coords). Nuevas tecnologías Comunicación Audiovisual y Educación (49-70). Barcelona: Dedes.

Green, A. D.; Brown, A.; Robinson, L. (2008). Making the most of the Web in your Classroom. A Teacher's guide to blog, podcasts, wikis, pages, and sites. Thousand, Oaks, CA, EEUU: Corwin Press.

Herrera, J. A.; Lozano, F. G.; Ramírez, M. S. (2008). Competencias aplicadas por los alumnos para el uso de dispositivos mlearning. Memorias del XVII Encuentro
Internacional de Educación a Distancia. Virtualizar para educar. Guadalajara, Jalisco, México.

Kenning, M-M. (2007). ICT and language learning from print to the mobile phone. New York, EEUU: Palgrave Macmillan.

Kukulska-Hulme, A.; Traxler, J. (2005). Mobile learning a handbook for educators and trainers. London, England: Routledge.

Laouris, Y. (2005). We need an Educationally Relevant Definition of Mobile Learning . [en línea] Disponible en: http://www. mlearn.org.za/papers-full.html[consulta 2007, 17 de Agosto]

Laouris, Y.; Eteokleous, N. (2005). We need an Educationally Relevant Definition of Mobile Learning. mLearn 2005 4th World conference on mLearning. Conference theme: Mobile technology: The future of learning in your hands. [en línea] Disponible en: http:// www.mlearn.org.za/CD/papers/ Laouris\%20\&\%20Eteokleous.pdf [consulta 2008, 17 de Octubre]

Lozano, F. G. (coord.) (2007). Competencias que requieren los alumnos para aprender con dispositivos m-learning. Documento interno, Tecnológico de Monterrey.

Marcos, L. M.; Tamez, R.; Lozano, A. (2008). M-learning como herramienta para el desarrollo de habilidades de debate en foros asincrónicos de comunicación. Memorias del Congreso Virtual Educa. Zaragoza, España.

Metcalf, D. S. (2006). mLearning: Mobile Learning and Performance in the Palm of your Hand. USA: HRD Press Inc. 
Recursos Tecnológicos Para El Aprendizaje Móvil (Mlearning) y su Relación con los Ambientes...

Montes, J.; Ochoa, S. (2006). Apropiación de las tecnologías de la información y la comunicación en cursos universitarios. Acta Colombiana de Psicología, 9, 2, (87-100).

Morales, S.; Monje, D.; Loyola, M. (2006). Apropiación crítica de NTIC's como camino de inclusión en la sociedad de la información: el caso de los jóvenes de una escuela secundaria de la ciudad de Córdoba. UNIrevista, 1, 3, (1-10).

Pastore, R. (2002). Elearning in Education: An Overview. Proceedings of Society for Information Technology and Teacher Education International Conference 2002. (275-276). Chesapeake, VA: AACE.

Pinkwart, N.; Hoppe, H. U.; Milrad, M.; Perez, J. (2003). Educational scenarios for the cooperative use of Personal Digital Assistant. Journal of Computer Assisted Learning, 19, 3, (383-391).

Quinn, C. (2000). mLearning: Mobile, wireless, in your-pocket learning. LiNE Zine. Fall.

Quinn, C. (2007). Mobile magic: Think different by design. Ciclo de conferencias dela Escuela de Graduados en Educación yCentrodeInnov@tedel Tecnológicode Monterrey, 18 de septiembre. Podcast [en línea] Disponible en: http://podcastuv. itesm.mx/ [consulta 2009, 2 de Julio]

Ramírez, M. S. (2008a). Dispositivos de mobile learning para ambientes virtuales: Investigación de implicaciones en el diseño y la enseñanza. Apertura Revista de Innovación Educativa, 9, (82-96).

Ramírez, M. S. (2008b). Implementación del mobile learning en los ambientes educativos. Memorias del $2^{\circ}$ Congreso Internacional de Elearning: Sin límites 2008 Trascendiendo fronteras. Bogotá, Colombia.
Ramírez, M. S. (2009). Inclusión del mobile learning en ambientes virtuales de aprendizaje. En: García-Valcárcel Muñoz-Repiso, A. (Ed.). Experiencias de Innovación Docente Universitaria (71-88). Ediciones Universidad de Salamanca.

Richardson, W. (2006). Blogs, wikis, podcasts, and the other powerful Web Tools for Classroom. Thousand, Oaks, CA, EEUU: Corwin Press.

Salz, P. A. (2005). When will we ever learn? Mobile Communications International, $1,129$.

Schrank,D.;Lomax,T.(2002).The20o2urban mobility report. Texas Transportation Institute. [en línea] Disponible en: http://tti.tamu.edu/documents/ums/ mobility report 2002.pdf [consulta 2008, 23 de Febrero]

Sharples, M. (2005). Learning as conversation: Transforming education in the mobile age. Proceedings "Seeing understanding, learning in the mobile age". Budapest, 28-30 de Abril, (147152).

Urrea, C. (2006). CREATE: Opportunities for Technology Appropriation. VIII Congreso Iberoamericano de Informática Educativa. Costa Rica.

Warschauer, M. (2003). Demystifying the digital divide. Scientific American, 289, $2,(42-71)$.

Young, J. R. (2002). Hybrid teaching seeks to end the divide between traditional and online. Chronicle of Higher Education, [en línea] Disponible en: http:// chronicle.com/free/v48/i28/28a03301. htm [consulta 2009, 2 de Julio] 


\section{PERFIL ACADÉMICO Y PROFESIONAL DE LA AUTORA}

María Soledad Ramírez Montoya. Profesora-investigadora titular de la Escuela de Graduados en Educación del Tecnológico de Monterrey. Es Doctora en Filosofía y Ciencias de la Educación por la Universidad de Salamanca (España). Desempeña actividades docentes, de investigación y de gestión a través de la dirección de la Cátedra de Investigación de Innovación en Tecnología y Educación. Es miembro del Sistema Nacional de Investigadores, teniendo como líneas de trabajo la enseñanza, los recursos tecnológicos y la educación a distancia.

E-mail: solramirez@itesm.mx

DIRECCIÓN DE LA AUTORA:

Av. Eugenio Garza Sada No. 2501 Sur, Edificio CEDES, Sótano 1

Col. Tecnológico Monterrey, Nuevo León 64849, México

Fecha de recepción del artículo: 09/03/09

Fecha de aceptación del artículo: 15/05/09 